\title{
Nociones numéricas de alumnos \\ mexicanos de tercero de preescolar
}

\section{Numerical notions of Mexican kindergarten pupils}

\author{
José Luis Cortina Morfín ${ }^{1}$ \\ Jesica Peña Jiménez ${ }^{2}$
}

\begin{abstract}
Resumen: Se analizan los resultados de un estudio en el que las nociones numéricas básicas de 22 alumnos que conformaban un grupo de tercero de preescolar - de una escuela pública mexicana- fueron evaluadas desde una perspectiva formativa. Todos eran hijos de familias viviendo en condiciones de pobreza. Los resultados muestran un rezago importante en el desarrollo de habilidades numéricas, en la gran mayoría de los estudiantes, cuando se toma como referencia a los aprendizajes que la autoridad educativa mexicana espera que alcancen los niños en el tercer grado de preescolar. Se discuten las implicaciones de nuestra investigación para la definición de una agenda pedagógica que apoye eficazmente el desarrollo matemático de niños como los evaluados, y cómo ésta se diferenciaría de la propuesta oficial actual.
\end{abstract}

Palabras clave: Educación Preescolar; Desarrollo Matemático; Numeración.

\begin{abstract}
We analyze a study in which the numerical skills of a class of Mexican kindergarten students were assessed $(\mathrm{N}=22)$. They were the children of families living in poverty. Findings show that almost all the children were lagging behind, when considering the mathematical learning goals for kindergarten in the Mexican curriculum. We discuss the implications of our findings for the defi-
\end{abstract}

Fecha de recepción: 26 de junio de 2017. Fecha de aceptación: 24 de mayo de 2018

${ }_{1}^{1}$ Universidad Pedagógica Nacional, Ajusco, jcortina@upn.mx, orcid,org/0000-0002-1926-0465

2 Universidad Pedagógica Nacional, Ajusco, jesica.pena.j@gmail.com, orcid.org/0000-0002-5396-1329 
nition of a pedagogical agenda that could effectively support the mathematical development of children like those that participated in the study, and how this agenda would differ from the current curriculum.

Keywords: Kindergarten; Mathematical Development; Numeration.

En México, desde el año 2004, la educación preescolar es obligatoria. La autoridad educativa -la Secretaría de Educación Pública (SEP)- emite programas de estudio en los que se incluyen temas de matemáticas. Además, se invierten importantes recursos en la edición de materiales educativos que reciben gratuitamente todos los alumnos y maestros. Sin embargo, la comunidad de investigación le ha prestado poca atención al aprendizaje de las matemáticas en preescolar. Se sabe muy poco sobre la pertinencia y efectividad de la educación matemática que se está realizando en este nivel, y sobre el impacto que tiene en el desarrollo matemático de los alumnos mexicanos.

Las causas de esto podrían ser múltiples y diversas, incluyendo el que las evaluaciones estandarizadas que se instrumentan a nivel internacional no circunscriben al nivel preescolar. Además, el tipo de actividad matemática a la que tienen acceso los niños pequeños puede parecer muy simple y de poca trascendencia para el aprendizaje de nociones relativamente complejas como la multiplicación, las fracciones y la proporcionalidad. Sin embargo, se ha identificado que existe una fuerte relación entre los niveles de desarrollo numérico que alcanzan los niños preescolares, y su desempeño matemático posterior, incluso después de controlar variables como la atención, y las habilidades cognitivas y socioemocionales (Duncan et al., 2007).

En un artículo publicado recientemente, Nguyen et al., (2016) reportan un estudio realizado en 42 escuelas de educación preescolar de los Estados Unidos, a las que asistían estudiantes de bajo nivel socioeconómico. Los investigadores evaluaron las habilidades matemáticas de 1,375 niños poco antes de ingresar al "kindergarten" (tercer grado de preescolar). Al paso de seis años, pudieron volver a evaluar a 781 de esos sujetos. Los investigadores encontraron que el nivel de desarrollo numérico que habían alcanzado los niños en el preescolar era un predictor muy robusto de su desempeño matemático en quinto de primaria. Los resultados de Nguyen y sus colegas corroboran lo que otros equipos 
de investigación ya habían encontrado (Geary, Hoard, Nugent, \& Bailey, 2013; Jordan, Kaplan, Ramineni, \& Locuniak, 2009).

En este artículo se reporta un estudio que consistió en evaluar las nociones numéricas que habían desarrollado 22 alumnos que conformaban un grupo de tercero de preescolar, de una escuela pública mexicana. Todos eran hijos de familias viviendo en condiciones de pobreza. La evaluación fue de naturaleza formativa (Scriven, 1967), en tanto que se diseñó con la intención de identificar los tipos de apoyos pedagógicos que les serían apropiados a niños como los evaluados, dado su nivel de desarrollo matemático.

Como se explica más adelante, nuestros resultados apuntan a la existencia de una brecha importante entre el nivel relativamente bajo de desarrollo de nociones numéricas, alcanzado por la enorme mayoría de los estudiantes evaluados, y el nivel que aparentemente deberían de tener, de acuerdo con la propuesta de la SEP para la enseñanza de las matemáticas para los tres grados de preescolar. Esta brecha es preocupante ya que implicaría que los esfuerzos pedagógicos que se realicen procurando seguir fielmente las recomendaciones de la SEP podrían resultar poco efectivos en apoyar el desarrollo matemático de estudiantes como los evaluados.

Comenzamos el artículo haciendo una revisión de la propuesta de la SEP para las matemáticas en preescolar. Después, explicamos la perspectiva pedagógica que orientó nuestro estudio, la cual se centra en las consideraciones de Clements y Sarama (2009) respecto a que la educación matemática en preescolar debe adecuarse al nivel de desarrollo de los niños. Posteriormente, explicamos la metodología empleada en la evaluación, describimos el tipo de situaciones que se utilizaron y presentamos los resultados obtenidos. Concluimos el artículo señalando las implicaciones de nuestra investigación para la definición de una agenda pedagógica que apoye eficazmente el desarrollo matemático de niños como los evaluados, y cómo ésta se diferenciaría de la propuesta actual de la SEP.

\section{EL NÚMERO EN EL PREESCOLAR MEXICANO}

La propuesta de la SEP para las matemáticas en preescolar está contenida en el programa de estudios para este nivel (SEP, 2011a) y en otros materiales que esta Secretaría le proporciona gratuitamente a alumnos y maestros. En ella se priorizan dos aspectos del pensamiento matemático: (1) número, y (2) forma, espacio y medida. Con respecto al primer aspecto, en el programa se destaca la 
importancia de que los estudiantes desarrollen habilidades numéricas básicas tales como: enunciar "una serie elemental de números en orden ascendente y descendente", usar "estrategias para contar", comprender "los principios del conteo", y resolver "problemas numéricos elementales en situaciones cotidianas" (p. 31).

Se espera que la formación matemática de los niños se origine en las actividades espontáneas e informales en que se implican, principalmente, fuera de la escuela. Según el programa de estudios de la SEP, el ambiente natural, cultural y social en que viven los niños "los provee de experiencias que, de manera espontánea, los llevan a realizar actividades de conteo" (SEP, 2011a, p. 51).

Se considera al conteo como la herramienta primordial para la resolución de problemas numéricos. A la vez, se concibe a la resolución de problemas como el medio principal a través del cual los niños desarrollarán la habilidad de contar, además de que los llevará a descubrir "las distintas funciones, usos y significados de los números" (SEP, 2011a, p. 56). Se espera que, a lo largo de todos los grados, los niños se involucren, de manera autónoma, en la resolución de situaciones que les sean familiares.

Es importante aclarar que el programa de estudios de la SEP no hace distinción de objetivos para los tres grados que incluye el nivel preescolar. De hecho, se autodefine como "de carácter abierto", por lo que "no presenta una secuencia de actividades o situaciones que deban realizarse sucesivamente con las niñas y los niños", sino que les delega a las educadoras la responsabilidad "de establecer el orden en que se abordarán las competencias propuestas para este nivel educativo, y seleccionar o diseñar las situaciones didácticas que considere(n) convenientes para promover las competencias y el logro de los aprendizajes esperados" (p. 15).

A pesar de ello, en otros materiales que la SEP le provee a docentes y alumnos, es posible reconocer expectativas sobre qué se debe enseñar en cada grado y de cómo debe ser enseñado. Los materiales principales son los libros Mi álbum, de los cuales existe una versión para cada uno de los tres grados que cubre el preescolar (SEP, 2014b, 2014c, 2014d). Además, está el Libro de la educadora (SEP, 2014a), el cual contiene propuestas didácticas para el trabajo con cada uno de los álbumes.

Las propuestas que se hacen en el Libro de la educadora para la enseñanza del número se centran en la resolución de problemas aditivos, los cuales van incrementando su complejidad en tres aspectos principales: el tamaño de las cantidades que entran en juego, las relaciones que se necesitan establecer, y las formas en que son representadas las cantidades. 
Para primer grado (niños de al menos dos años y ocho meses de edad al iniciar el año escolar), el Libro de la educadora recomienda promover el desarrollo de habilidades básicas de conteo a través de pedirle a los alumnos que determinen la cantidad que hay en colecciones de hasta seis elementos. También, se espera que resuelvan problemas de comparación simples como determinar cuál de dos fichas de dominó tiene más puntos.

Para segundo grado (niños de tres y cuatro años), se espera que los estudiantes trabajen con colecciones de hasta ocho elementos, además de que resuelvan problemas que implican establecer diferentes tipos de relaciones cuantitativas. Por ejemplo, una de las láminas del álbum contiene el dibujo de un tapete en el que se encuentran desordenadamente distribuidos 47 juguetes, de doce diferentes tipos: banderines (7), canicas (6), capas de luchador (6), tortugas (5), luchadores (4), matatenas (4), coches (4), trompos (4), yoyos (3), baleros (2), camioneta (1) y cuerda para trompo (1). El Libro de la educadora propone aprovechar la lámina para presentar problemas que implican comparar, igualar, agregar, quitar, repartir, y reunir. En la Tabla 1 se muestra un ejemplo de cada uno.

Tabla 1. Ejemplos de seis diferentes tipos de problemas numéricos simples tomados del Libro de la educadora (SEP, 2014a).

\begin{tabular}{|l|}
\hline \multicolumn{1}{|c|}{ Ejemplos de problemas numéricos } \\
\hline ¿Qué hay más: luchadores o coches? \\
\hline ¿Qué podríamos hacer para tener la misma cantidad de baleros y de canicas? \\
\hline Jorge tiene un coche más de los que hay en la lámina. ¿Cuántos coches tiene Jorge? \\
\hline Valentina tiene una tortuga menos de las que hay en la lámina. ¿Cuántas tortugas tiene? \\
\hline $\begin{array}{l}\text { Jorge y Valentina van a jugar con los trompos. ¿Cuántos tendrá cada uno si se los reparten y } \\
\text { les toca la misma cantidad? }\end{array}$ \\
\hline Si llega una persona y compra todas las canicas, ¿cuántas se lleva? \\
\hline
\end{tabular}

También para segundo grado, se espera que los estudiantes resuelvan problemas que implican darle una interpretación cardinal a los números escritos, siempre en contextos de dinero. Por ejemplo, en una de las láminas se presenta el dibujo de un puesto de frutas. Al frente de cada conjunto de frutas aparece el precio unitario de cada fruta. Los precios oscilan entre "1 peso" (limones, fresas y 
ciruelas) y "9 pesos" (sandías). Las sugerencias de problemas que se hacen en el Libro de la educadora se centran en sumar el precio de dos productos, como en estos ejemplos: “¿Cuánto tienen que pagar por una manzana [\$5] y un limón [\$1]?, ¿̇cuánto por una piña [\$8] y una fresa [\$1]?, ¿̇cuánto por un aguacate [\$5] y una pera [\$3]?". Se recomienda proveer a los niños de múltiples monedas de juguete de un peso, cuando se les pida que resuelvan estos problemas.

Para tercer grado, los tipos de problemas son, también, de comparar, igualar, agregar, quitar, repartir, y reunir, pero implican trabajar con cantidades mayores. Además, se incrementa notablemente la presencia de problemas en los que se necesita darle una interpretación cardinal a los números escritos. También se incrementa su complejidad. Por ejemplo, se espera que los niños resuelvan problemas que implican relacionar el valor de monedas con cuatro denominaciones diferentes: $\$ 1, \$ 2, \$ 5$ y $\$ 10$.

Al egresar del preescolar, se espera que los estudiantes hayan adquirido habilidades numéricas relativamente complejas, entre las que se incluyen las capacidades de utilizar números naturales hasta de dos cifras para interpretar o comunicar cantidades, y de resolver problemas aditivos simples, usando representaciones gráficas o el cálculo mental, pudiendo explicar qué se hizo para resolverlos (SEP, 2011a). Es importante mencionar que estas expectativas son consistentes con los programas de estudio de primaria (SEP, 2011b), en los que se espera que los niños se impliquen, al iniciar el primer grado, en la resolución de problemas que requieren agregar o quitar elementos a una colección, juntar o separar colecciones, buscar lo que le falta a una cierta cantidad para llegar a otra, y avanzar o retroceder en una sucesión, con resultados menores a 30.

\section{ORIENTACIÓN PEDAGÓGICA}

La evaluación se diseñó tomando como base las consideraciones de Clements y Sarama (2009) respecto a la educación matemática eficaz en el nivel preescolar. Estos autores identifican dos estrategias pedagógicas distintas para procurar el logro de objetivos de aprendizaje ambiciosos. La primera implica la instrumentación de un tipo de enseñanza que se enfoque directamente en los aprendizajes esperados, lo que hace que se pasen por alto los niveles de desarrollo de los niños. Por ejemplo, si se espera que los estudiantes aprendan a resolver problemas aditivos simples, se procura involucrarlos, constantemente, en la reso- 
lución de este tipo de problemas, independientemente del nivel de desarrollo que hayan alcanzado en el campo numérico.

La segunda estrategia reconocida por Clements y Sarama (2009) es la que sirvió de base para el diseño de la evaluación y, de acuerdo con los resultados preliminares de una investigación en curso (Clements, Baroody, \& Sarama, Abril, 2017), es la que puede resultar mucho más eficaz. En ella se procura que la enseñanza se adecue al nivel de desarrollo matemático de los niños, dentro de una trayectoria de aprendizaje. Es así que las actividades que impliquen directamente a los aprendizajes esperados no se usan sino hasta que se considera que los estudiantes ya han desarrollado las nociones mínimas necesarias para encontrarles pleno sentido. Antes, se procura que las actividades de enseñanza sean apropiadas para el nivel en que se encuentren los niños. Además, se recomienda que estas actividades les resulten retadoras a los niños, sin llegar al punto de que no les sean asequibles (Clements, 2004).

Por supuesto, la instrumentación de la segunda estrategia requiere de conocer el nivel de desarrollo matemático de los estudiantes. Sin eso, no sería posible definir qué tipo de actividades de enseñanza les resultarían más adecuadas.

\section{METODOLOGÍA}

Para realizar el estudio se hizo una adaptación de la metodología propuesta por McGatha, Cobb, y McClain (2002), para identificar puntos de partida para el diseño didáctico. La metodología de estos autores involucra el diseño y aplicación de actividades, que tienen la finalidad de evaluar formativamente el desempeño de grupos completos de alumnos (whole-class performance assessment tasks), lo que incluye identificar su nivel general de comprensión de una noción matemática en particular.

En la adaptación que se hizo de la metodología, las actividades fueron aplicadas en grupos pequeños de estudiantes, en lugar de un grupo completo; ello, con la finalidad de poder recabar información más o menos detallada de cada uno de los alumnos, en relación con su nivel de desarrollo en el campo del pensamiento numérico.

Las actividades se aplicaron a los 22 alumnos que conformaban un grupo de tercero de preescolar, de una escuela pública mexicana. La aplicación se hizo en el mes de noviembre, cuando todos los niños tenían cinco años de edad y 
había transcurrido aproximadamente una quinta parte del año escolar. Para evaluar a los alumnos se les organizó en seis grupos pequeños, de los cuales cinco estuvieron formados por cuatro estudiantes y uno por dos. De los 22 alumnos, quince eran niñas.

La segunda autora de este artículo fue quien se encargó de instrumentar la evaluación de todos los niños. Trabajó con los alumnos que formaban cada grupo, sentando en una mesa a dos estudiantes a su derecha y a dos a su izquierda. Mientras los alumnos de cada grupo fueron evaluados, el resto de los niños trabajó con su maestra, de manera regular. La evaluación de los alumnos de cada grupo pequeño duró aproximadamente 50 minutos.

\section{LA TRAYECTORIA DE APRENDIZAJE}

En la literatura en el campo se reconoce al conteo como un aspecto central del pensamiento numérico de los niños pequeños (para una revisión profunda de la literatura en el tema ver Sarama \& Clements, 2009). Aprender a contar requiere del desarrollo de múltiples habilidades mentales, las cuales han sido el foco de muchos trabajos a lo largo de varias décadas. Aunque no existe un consenso generalizado respecto a cuáles son éstas, se considera que una muy importante es la capacidad de concebir a la numerosidad que se reconoce al contar una colección, como un atributo que no desaparece al concluir el conteo, sino que se conserva y caracteriza a una propiedad del conjunto; esto es, la cantidad de elementos que tiene (Steffe \& Cobb, 1988).

El término con el que comúnmente se le denomina a este aspecto del conteo es cardinalidad. Según Gelman y Gallistel (1978), el conteo con cardinalidad conlleva entender que la última etiqueta utilizada en la enumeración de un conjunto tiene un significado especial, una vez que no sólo etiqueta al último elemento, sino que también representa al total de elementos que hay en la colección.

Sarama y Clements (2009) reconocen a la cardinalidad como la cúspide del desarrollo numérico temprano, y la base de todo el desarrollo posterior en el campo de las operaciones numéricas. Esto se debe a que la adquisición del conteo con cardinalidad es necesario para darle sentido a situaciones que implican operar con una cantidad; por ejemplo, comparando su tamaño con el de otra, determinando su tamaño después de agregarle o quitarle elementos, o el tamaño de los conjuntos que se forman después de repartir una cantidad de elementos en partes iguales. 
Según Baroody (2000), para desarrollar el conteo con cardinalidad, los niños primero deben lograr enumerar correctamente; esto es, establecer una correspondencia uno a uno entre los números que secuencialmente pronuncian y los elementos de una colección, de manera que a todos y cada uno de los elementos se le asigne una etiqueta numérica y sólo una. Antes, todavía, deben de lograr enunciar la serie numérica de manera estable y correcta.

Es entonces posible identificar los elementos básicos de una trayectoria general de aprendizaje del conteo temprano, la cual comienza con el dominio de la serie numérica oral inicial (también conocido como conteo oral), continúa con la enumeración de colecciones pequeñas, y culmina con la cardinalidad.

En el diseño de la evaluación se procuró incluir situaciones que ayudaran a conocer el dominio de los niños de los tres componentes de la trayectoria de aprendizaje antes mencionada. Además, se evaluaron otros dos aspectos que, como se explica más adelante, se consideran centrales en el desarrollo del pensamiento numérico temprano: el conocimiento de los numerales escritos hasta el diez, y la subitación de hasta seis elementos. La forma en que se evaluó el dominio de cada uno de estos elementos se describe a continuación.

\section{DOMINIO DE LA SERIE NUMÉRICA ORAL}

El dominio de la serie numérica oral hasta el diez fue evaluado a través de un juego que denominamos "Ping-Pong". En él se utilizó una pelota pequeña. Se inició evaluando el dominio de la serie cuando se enuncia en orden ascendente. La evaluadora les explicó a los niños algo similar a lo siguiente: "Yo voy a decir el primer número de la serie numérica. El niño al que le dé la pelota va a decir el siguiente número. Me regresa la pelota y yo diré el número que continúa. Entonces, le daré la pelota a otro niño y él dirá el número que sigue. Así, hasta llegar al diez". Si alguno de los niños decía un número equivocado, la evaluadora mencionaba el número correcto y continuaba el juego sin señalar el error.

El juego tuvo algunas variantes para identificar mejor el nivel de dominio de la serie numérica oral de cada niño. Una de ellas fue que los niños se pasaran la pelota entre ellos diciendo los números, mientras la evaluadora sólo observaba. Además, si después de haber jugado varias veces, la evaluadora aún tenía dudas respecto hasta qué número dominaba alguno de los niños, ella jugaba exclusivamente con él, mientras los demás observaban. 
Para evaluar la serie numérica oral en orden descendente, se les dio a los niños una indicación similar a la siguiente: "Ahora, vamos a decir la serie numérica oral en orden descendente. La vamos a decir del diez al uno. Yo iniciaré y le daré la pelota a un niño para que diga el número que va antes del que dije".

Cuando se identificó que a uno o varios niños se les dificultaba el juego comenzando en diez, se replanteó la actividad para que sólo se enunciara la serie del cinco hacia atrás.

Por último, se trató de identificar si los niños podían mencionar el antecesor y el sucesor de diferentes números entre el uno y el diez. Se les dieron explicaciones similares a éstas: "Ahora, yo voy a decir un número y ustedes mencionarán el número que sigue". "Ahora, yo voy a decir un número y ustedes mencionarán el número que va antes".

En esta parte de la evaluación se procuró decirles a los niños números que estuvieran incluidos en la serie que habían pronunciado correctamente. Por ejemplo, a los niños que sólo enunciaron la serie numérica de manera correcta hasta el cuatro, no se les preguntó por el sucesor o antecesor del seis.

\section{ENUMERACIÓN DE COLECCIONES Y CARDINALIDAD}

Para evaluar la enumeración de colecciones y el conteo con cardinalidad se diseñó una actividad que llamamos “¿Cuántos son?", en la que se usaron 18 platos de cartón a los que se les adhirieron dulces. Los platos tuvieron entre dos y diez dulces. En la mitad de los platos, los dulces estuvieron alineados y en la otra mitad no (ver Figura 1).

Para evaluar la enumeración, a los estudiantes se les dieron explicaciones similares a éstas: "A cada uno le voy a dar un plato con dulces. Los dulces están pegados. No se pueden despegar. A la cuenta de tres todos van a voltear su plato y me van a decir cuántos dulces les tocaron".

Al frente de cada estudiante se colocó uno de los platos boca abajo para que no vieran la cantidad de dulces. Primero se usaron los platos que tenían entre dos y cinco dulces no alineados. Se les dio tiempo suficiente a los niños para que contaran el número de dulces que tenían en el plato que les tocó. Se puso atención en si los estudiantes contaban la colección de dulces estableciendo una correspondencia uno a uno con la serie numérica que pronunciaban. 

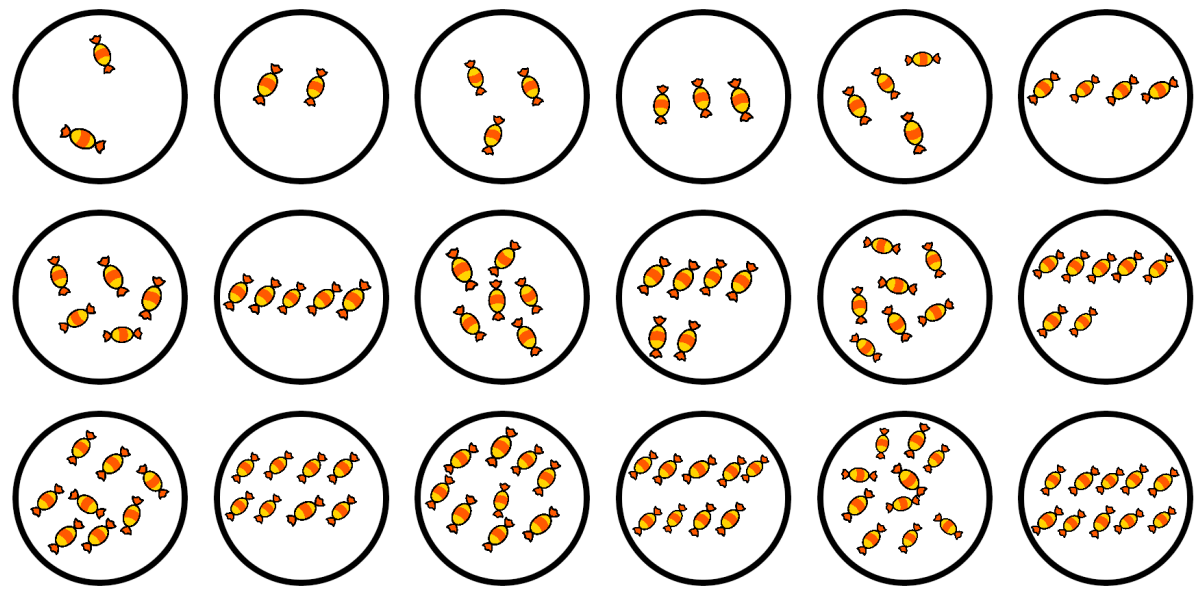

Figura 1. Dulces organizados y desorganizados en los platos de cartón.

A los niños que enumeraban correctamente se les daba un plato con un número mayor de dulces, hasta identificar hasta qué cantidad podían enumerar correctamente. A los niños que no enumeraban correctamente, se les daba un plato que fuera más fácil de enumerar, ya fuera por tener los dulces alineados o por tener menos dulces.

Una forma en que se evaluó el conteo con cardinalidad, consistió en preguntarle a los niños cuántos dulces había en su plato, después de que ya habían enumerado la colección. A quienes volvían a enumerar, en lugar de repetir el último número que habían contado, se les consideraba como que no habían contado con cardinalidad.

Además, la evaluadora colocó un dulce más en los platos que ya habían sido contados y les pidió a los niños que le dijeran ahora cuántos dulces había. A los niños que respondían diciendo el siguiente número en la serie, y que no tenían necesidad de enumerar el conjunto como si se tratara de una nueva colección, se les consideraba que ya habían desarrollado la noción de cardinalidad; al menos para contar colecciones con la cantidad de elementos que había en el plato.

La actividad se realizó hasta que la evaluadora se formó una idea clara del tamaño de colecciones que cada niño podía enumerar, estando alineadas y desalineadas, y de quiénes contaban con cardinalidad. 


\section{SUBITIZAR}

La subitización fue otro aspecto del pensamiento numérico temprano que se evaluó. Ésta consiste en reconocer de manera inmediata la cantidad de elementos que hay en un conjunto pequeño. En el programa de estudios de la SEP se le reconoce como una competencia a desarrollar, y se le define como: "[el alumno] Identifica por percepción, la cantidad de elementos en colecciones pequeñas" (SEP, 2011a, p. 57)

Según autores como Baroody (2000) y Sarama y Clements (2009), la subitización es fundamental en el desarrollo de concepciones numéricas, una vez que se apoya en el reconocimiento de patrones, y en el descubrimiento de propiedades numéricas esenciales, como la conservación, la composición y la compensación.

La habilidad de los niños para subitizar se evaluó a través de una actividad que llamamos “¿Cuántos viste?". En ella se utilizaron doce platos de cartón en los que se adhirieron estampas circulares a las que se les llamó "puntos". Los platos tuvieron entre uno y seis puntos distribuidos de diferente manera (ver Figura 2).

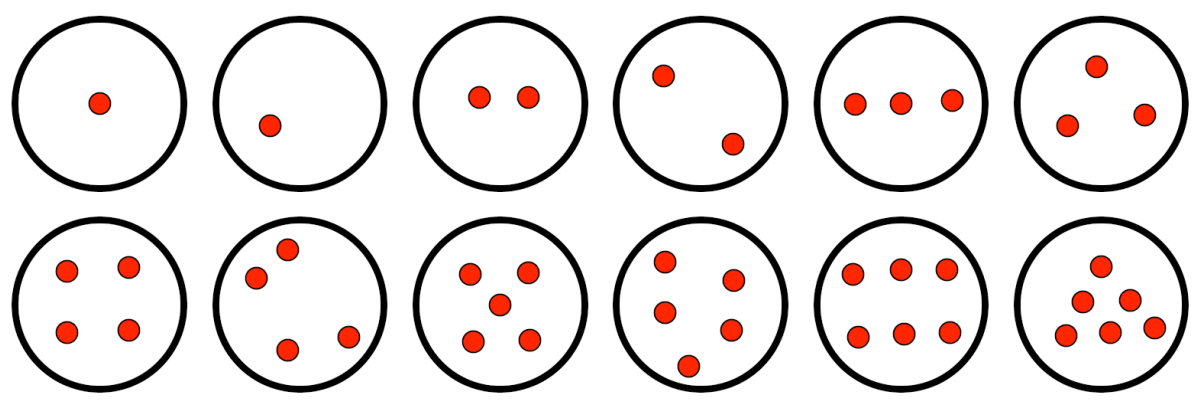

Figura 2. Configuraciones en las que se organizaron los puntos en los platos de cartón

A los estudiantes se les dieron explicaciones similares a éstas: "Les voy a enseñar unos platos con puntos, pero no se vale decir cuántos puntos vieron hasta que yo les pregunte". Además, se les enfatizó a los niños que sólo debían responder cuando la evaluadora les preguntara directamente. 
Primero se les mostró a los niños un plato con uno, dos o tres puntos. El tiempo en el que se le exhibió cada plato fue suficiente para que los niños vieran los puntos, pero no para que los contaran (dos o tres segundos).

A continuación, se le preguntó a cada niño cuántos puntos había visto. Conforme se desarrolló la actividad, a los niños que respondían correctamente, se le fueron presentando platos con configuraciones difíciles de subitizar. A los niños que no acertaban, se les mostraron platos más fáciles de identificar, ya sea porque tenían menos puntos o porque los puntos estaban ordenados.

\section{LECTURA DE NÚMEROS}

El último aspecto del desarrollo numérico temprano que evaluamos fue la lectura de números. Según Sarama y Clements (2009), el uso de los números escritos para representar cantidades es importante en la ruta hacia el pensamiento matemático abstracto. En el programa de estudios de la SEP (2011a), la lectura de números se incluye en las competencias a desarrollar: "Identifica el orden de los números en forma escrita, en situaciones escolares y familiares" (p. 57).

Para evaluar este aspecto, se usó un juego que llamamos “¿Qué Número Es?". En él se usaron tarjetas (de $10 \mathrm{~cm}$ por $10 \mathrm{~cm}$ ) con los números escritos del 1 al 10. En cada tarjeta se escribió un número (ver Figura 3).
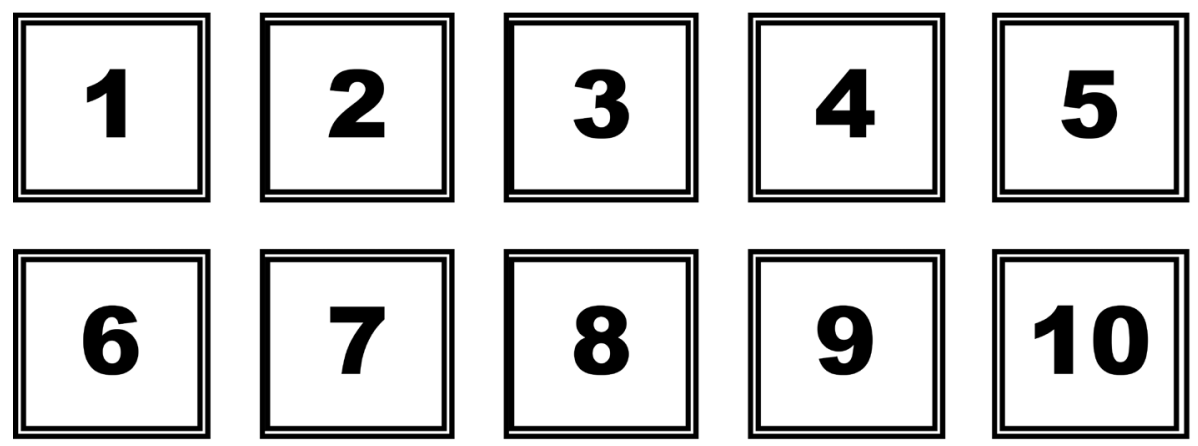

Figura 3. Tarjetas con números escritos que fueron usadas 
A los estudiantes se les dieron explicaciones similares a éstas: "Voy a mostrar algunos números. Sólo me va a responder 'qué número es' el niño al que yo le pregunte".

Primero se mostraron al azar las tarjetas con los números del 1 al 5. Si se consideraba necesario, una tarjeta era presentada varias veces para verificar si el número era identificado correctamente (o no) por todos niños. A los niños que identificaban correctamente los números del 1 al 5, se les mostraban las tarjetas con los números escritos del 6 al 10.

La actividad continuó dándole a cada niño un conjunto de tarjetas con los numerales y pidiéndoles que las pusieran en orden. La evaluadora procuró incluir en el conjunto que recibía cada niño únicamente tarjetas que hubiera identificado correctamente.

\section{ANÁLISIS DE DATOS}

Para analizar los resultados se creó una tabla de doble entrada en la que se registró de manera sucinta el desempeño de cada niño en la evaluación, incluyendo:

El número hasta el que enunció correctamente la serie numérica en orden ascendente

El número desde el que enunció correctamente la serie numérica en orden descendente

El número hasta el que reconoció su antecesor y sucesor con facilidad

El número máximo de elementos en una colección que enumeró con facilidad

El número máximo de elementos en una colección que contó con cardinalidad

El número máximo de puntos que subitizó correctamente

Los números escritos que leyó correctamente

Los números escritos que ordenó correctamente

Debido a su doble entrada, la tabla permitió identificar fácilmente la diversidad en el desempeño de los niños en cada ítem, así como el desempeño que cada individuo tuvo en todos los ítems. 


\section{RESULTADOS}

Con base en su desempeño, se identificaron tres grupos de alumnos. El de mejor desempeño (Grupo 3; ver Tabla 2) estuvo conformado por cuatro alumnos, dos niños y dos niñas, que parecieron haber desarrollado las habilidades necesarias para contar colecciones de al menos diez elementos con cardinalidad.

Tabla 2. Distribución de los alumnos evaluados por grupos, de acuerdo con su desempeño.

\begin{tabular}{|l|c|c|c|c|c|}
\hline & \multicolumn{2}{|c|}{$\begin{array}{c}\text { Grupo 1 } \\
\text { Contadores iniciales }\end{array}$} & \multicolumn{2}{|c|}{$\begin{array}{c}\text { Grupo 2 } \\
\text { Enumeradores }\end{array}$} & \multicolumn{2}{|c|}{$\begin{array}{c}\text { Grupo 3 } \\
\text { Contadores con } \\
\text { cardinalidad }\end{array}$} & Total \\
\hline $\begin{array}{l}\text { Núm. de } \\
\text { Alumnos }\end{array}$ & 4 & 3 & 11 & 4 & 22 \\
\hline Porcentaje & 18.2 & 13.6 & 50.0 & 18.2 & 100.0 \\
\hline
\end{tabular}

Ellos pudieron enunciar con facilidad de manera ascendente y descendente la serie numérica hasta el diez. Además, pudieron identificar, casi sin dudar, el antecesor y sucesor de cualquier número que se les preguntara. Cuando enumeraron, lo hicieron estableciendo una correspondencia uno a uno en colecciones de hasta diez dulces. Cuando se les preguntó cuántos dulces había, siempre contestaron repitiendo el último número que contaron. Además, cuando se agregó un dulce más a la colección, no volvieron a enumerar, sino que respondieron diciendo el siguiente número de la serie. También subitizaron correctamente todas las colecciones de puntos (ver Figura 2), leyeron todos los números escritos y ordenaron correctamente las tarjetas.

El segundo grupo identificado se conformó por 14 alumnos, 12 niñas y 2 niños (Grupo 2; ver Tabla 2) que no mostraron haber desarrollado aún el conteo con cardinalidad, pero sí tener cierto dominio de la enumeración. Hubo diferencias en las habilidades que mostraron, lo que nos llevó a ubicar a los niños de este grupo en dos subgrupos.

Los alumnos del subgrupo B (ver Tabla 2) fueron los que se desempeñaron mejor. Enunciaron correctamente la serie numérica oral en orden ascendente del uno al diez. De manera descendente pudieron decirla correctamente sólo del 
cinco al uno. Para reconocer el antecesor y sucesor de los números del uno al diez tuvieron que decir la serie desde el principio y, aun así, no les resultó fácil identificarlos.

Enumeraron correctamente colecciones de hasta ocho dulces. Con colecciones de nueve y diez dulces no mantuvieron la correspondencia uno a uno. Cuando se les preguntó cuántos dulces había, siempre volvieron a contar la colección completa. Lo mismo hicieron cuando se les colocó un dulce más a una colección que ya habían enumerado.

Pudieron subitizar colecciones de hasta cuatro puntos. Respecto a los números escritos, los niños de este subgrupo identificaron y ordenaron correctamente del uno al cinco. No reconocieron los números del seis al diez.

El desempeño de los estudiantes ubicados en el otro subgrupo (subgrupo A; ver Tabla 2) fue más pobre. Al igual que sus compañeros de grupo, enunciaron correctamente la serie numérica oral en orden ascendente del uno al diez. Sin embargo, no pudieron enunciar la serie de manera descendente, ni siquiera del cinco al uno. Enumeraron colecciones de hasta seis elementos con correspondencia uno a uno. Cuando se les preguntó cuántos había, siempre volvieron a enumerar la colección. Lo mismo hicieron cuando se les colocó un dulce más a una colección que ya habían enumerado. Sólo leyeron correctamente algunos números; por ejemplo: 1, 2, y 5. Súbitamente identificaron colecciones de hasta tres puntos.

El tercer y último grupo identificado (Grupo 1; ver Tabla 2) se conformó por cuatro estudiantes, tres niños y una niña, que parecieron estar en una etapa bastante temprana de desarrollo numérico. Estos estudiantes enunciaron correctamente la serie numérica oral en orden ascendente sólo hasta el tres. Enumeraron colecciones de hasta tres elementos, únicamente. Cuando se les daba un plato con más de tres dulces señalaban y decían números de manera que parecía aleatoria. Cuando se les peguntaba: “¿Cuántos dulces tienes?", volvían a enumerar, decían lo que parecía un número al azar, o se quedaban callados. Sólo identificaron el número 1 de manera escrita. Súbitamente, sólo reconocieron configuraciones con dos puntos.

\section{INTERPRETACIÓN}

Con base en las consideraciones de Clementes y Sarama (2009), en el sentido de que la enseñanza en el nivel preescolar debe estar adecuada al nivel de desarrollo 
matemático de los niños; los resultados nos muestran un grupo con alumnos para quienes, en su gran mayoría, las actividades que la autoridad mexicana recomienda que se prioricen en segundo y tercero de preescolar aún no serían pertinentes. Como se explicó, sólo los cuatro alumnos ubicados en el Grupo 3 parecían haber desarrollado el conteo con cardinalidad y, por lo tanto, estar listos para involucrarse plenamente en la resolución de problemas aditivos sencillos. Estos estudiantes quizá, también, estarían listos para involucrarse en situaciones que implicaran atribuirle un significado cardinal a los números escritos.

Para los catorce alumnos ubicados en el Grupo 2, problemas que implicaran comparar la cantidad de elementos en dos colecciones de objetos, igualar su tamaño, agregarle o quitarle cierta cantidad de elementos a una colección, parecerían no serles aún asequibles. Estos niños no sólo no habían desarrollado aún la habilidad de contar con cardinalidad, sino que tampoco parecieron tener un dominio cabal de la enumeración de colecciones pequeñas y de la serie numérica oral inicial. Además, parecieron estar poco familiarizados con los números escritos.

A estos alumnos, actividades lúdicas enfocadas a apoyar el dominio de la serie numérica, de la enumeración, y la lectura de los primeros números, les podrían resultar retadoras y provechosas. Vale la pena señalar que estas actividades serían significativamente más simples que las que recomienda la SEP, incluso para segundo grado de preescolar (ver arriba).

En cuanto a los cuatro alumnos ubicados en el Grupo 1, situaciones que implicaran operar con la cantidad de elementos que tiene una colección parecerían estar completamente fuera de su alcance inmediato. Conjeturamos que el desarrollo de nociones numéricas de estos niños se beneficiaría si se involucraran en actividades que implicaran enunciar los primeros números de la serie oral y enumerar colecciones con muy pocos elementos. Creemos que también resultarían provechosas actividades lúdicas que implicaran la subitización de colecciones pequeñas y la lectura de los números dígitos.

\section{DISCUSIÓN Y CONCLUSIONES}

En conjunto, los resultados muestran a un grupo de alumnos con un nivel de desarrollo numérico significativamente más bajo del que, presumiblemente, los creadores de los recursos pedagógicos que edita la SEP esperan que tengan no sólo quienes cursan el tercer grado de preescolar, sino también el segundo. Con base en los estudios citados al principio del presente artículo (Duncan et al., 2007; 
Geary et al., 2013; Jordan et al., 2009; Nguyen et al., 2016), nuestros resultados indican que el aprendizaje matemático a largo plazo de la gran mayoría de los niños en este grupo podría estar en grave riesgo, de no recibir apoyos que les permitieran avanzar rápida y sustancialmente en su comprensión numérica.

Desafortunadamente, estos apoyos probablemente no se harían realidad si la enseñanza matemática que se le procurara se apegara a las recomendaciones que hace la SEP, sobre todo en el llamado Libro de la educadora (SEP, 2014a). Como ya se explicó, el tipo de situaciones que ahí se recomienda emplear no estarían adecuadas al nivel de desarrollo numérico mostrado por la gran mayoría de los estudiantes evaluados; por lo que, con base en la orientación pedagógica adoptada en el presente artículo, podrían resultar poco fructíferas.

Consideramos que el aparente rezago de la mayoría de los niños evaluados estaría relacionado con la situación social, familiar y económica en que vivían. Todos eran hijos de familias socioeconómicamente desfavorecidas. En general, el ingreso familiar provenía de alguna actividad mal remunerada, como el servicio doméstico o el comercio informal. La escolaridad de la mayoría de los padres era baja, y no contaban con fácil acceso a servicios de salud de calidad.

Nuestra consideración es consistente con el extenso cuerpo de literatura que reconoce que existe una fuerte relación entre el desempeño matemático de los alumnos y las circunstancias económicas, culturales y sociales en que viven sus familias (ej., Backhoff, Bouzas, Hernández, \& García, 2007). También lo es con investigaciones que han reconocido que los ambientes domésticos de los niños que viven en situación de pobreza son de menor riqueza matemática que los de aquellos que viven en mejores circunstancias (Siegler, 2009).

Conjeturamos entonces que, para el caso del desarrollo de nociones numéricas tempranas de niños mexicanos como los evaluados, el ambiente en que viven sería relativamente pobre en términos de experiencias matemáticas; al menos del tipo que lleven a los niños pequeños a involucrarse de manera informal en actividades como en el conteo, la cuantificación de conjuntos y el uso de los números escritos.

En términos pedagógicos, de ser válida nuestra conjetura, implicaría que el desarrollo de habilidades numéricas básicas de alumnos como los evaluados, dependería fuertemente de las oportunidades que les proporcionara la escuela. Siguiendo la orientación pedagógica aquí adoptada, esas oportunidades serían mejores si las actividades de enseñanza se adecuaran al nivel de desarrollo de los niños. 
Es importante aclarar que, evidentemente, nuestro estudio no implicó el uso de una muestra estadísticamente representativa que permitiera inferir la presencia relativa de estudiantes como los evaluados en el alumnado mexicano. Sin embargo, nuestros resultados son, en términos generales, consistentes con los de las evaluaciones realizadas por el Instituto Nacional de Evaluación de la Educación (INEE). Por ejemplo, en la aplicación de 2011 de los Exámenes de la Calidad y el Logro Educativos (INEE, 2014) se detectó que, al concluir el preescolar, 70\% de los estudiantes preescolares mexicanos con "capital económico familiar bajo" no eran capaces de "resolver problemas que implican agregar, igualar, comparar, quitar o repartir cantidades de una o más colecciones de hasta nueve objetos" (p. 44).

Además, no se debe perder de vista que en México la mayoría de los niños viven en situación de pobreza (Consejo Nacional de Evaluación de la Política de Desarrollo Social, 2016). Sería entonces razonable esperar que hubiera un importante número de estudiantes de tercero de preescolar cuya situación escolar, relativa al aprendizaje matemático, fuera muy similar a la de los alumnos evaluados.

Quedaría pendiente establecer qué tan beneficiosa resultaría ser una enseñanza que se adecuara al nivel de desarrollo matemático de niños como los evaluados, y, si sería suficiente para que alcanzaran los niveles que la SEP espera que tengan quienes están a punto de ingresar a la educación primaria. En nuestra investigación en curso estamos explorando estas cuestiones. También consideramos que sería importante indagar hasta qué punto una enseñanza matemática pertinente y eficaz, en el nivel preescolar, impactaría positivamente en el desempeño matemático a largo plazo de estudiantes como los evaluados.

\section{REFERENCIAS}

Backhoff, E., Bouzas, A., Hernández, E., \& García, M. (2007). Aprendizaje y desigualdad social en México. Implicaciones de política educativa en el nivel básico. México, D.F.: Instituto Nacional para la Evaluación de la Educación.

Baroody, A. J. (2000). El pensamiento matemático de los niños: Un marco evolutivo para maestros de preescolar, ciclo inicial y educación especial. Madrid: Visor.

Clements, D. H. (2004). Major themes and recommendations. In D. H. Clements, J. Sarama, \& A. DiBiase (Eds.), Engaging young children in mathematics: Standards for early childhood mathematics education. Mahwah, Nueva Jersey, EUA: Lawrence Erlbaum. 
Clements, D. H., Baroody, A. J., \& Sarama, J. (Abril, 2017). Evaluating the efficacy of learning trajectories in early math-Experiment 1. Paper presented at the National Council of Teachers of Mathematics Research Conference, San Antonio, Texas, EUA.

Clements, D. H., \& Sarama, J. (2009). Learning and teaching early math: The learning trajectories approach. Nueva York: Routledge.

Consejo Nacional de Evaluación de la Política de Desarrollo Social. (2016). Informe de pobreza en México, 2014. Ciudad de México: Autor.

Duncan, G. J., Dowsett, C. J., Claessens, A., Magnuson, K., C., H. A., Pamela, K., ... Japel, C. (2007). School readiness and later achievement. Developmental Psychology, 43(6). doi:10.1037/0012-1649.43.6.1428

Geary, D. C., Hoard, M. K., Nugent, L., \& Bailey, D. H. (2013). Adolescents' functional numeracy is predicted by their school entry number system knowledge. PLoS One, 8(1), 1-8. doi:10.1371/journal.pone.0054651

Gelman, R., \& Gallistel, C. R. (1978). The child's understanding of number. Cambridge, Massachusetts, EUA: Harvard University Press.

Instituto Nacional para la Evaluación de la Educación. (2014). El aprendizaje en preescolar en México. Informe de resultados EXCALE 00 aplicación 2011. Lenguaje y comunicación y pensamiento matemático. México, D.F.: Author.

Jordan, N. C., Kaplan, D., Ramineni, C., \& Locuniak, M. N. (2009). Early math matters: kindergarten number competence and later mathematics outcomes. Developmental Psychology, 45(3), 850-867. doi:10.1037/a0014939

McGatha, M., Cobb, P., \& McClain, K. (2002). An analysis of students' initial statistical understandings: Developing a conjectured learning trajectory. Journal of Mathematical Behavior, 16(3), 339-355. doi:10.1016/S0732-3123(02)00133-5

Nguyen, T., Wattsa, T. W., Duncana, G. J., Clements, D. H., Sarama, J. S., Wolfe, C., \& Spitler, M. E. (2016). Which preschool mathematics competencies are most predictive of fifth grade achievement? Early Childhood Research Quarterly, 36, 550-560. doi:10.1016/j.ecresq.2016.02.003

Sarama, J., \& Clements, D. H. (2009). Early childhood mathematics education research: Learning trajectories for young children. New York: Routledge.

Scriven, M. (1967). The methodology of evaluation. Perspectives of Curriculum Evaluation. American Educational Research Association. Monograph on Curriculum Evaluation Num. 1. Chicago: Rand McNally.

Secretaría de Educación Pública. (2011a). Programa de estudio 2011. Guía para la educadora. Educación básica. Preescolar. México, D.F.: Autor.

Secretaría de Educación Pública. (2011b). Programas de estudio 2011. Guía para el Maestro. Educación Básica. Primaria. Primer grado. México, D. F.: Author. 
Secretaría de Educación Pública. (2014a). Libro de la educadora. México, D. F.: Autor.

Secretaría de Educación Pública. (2014b). Mi álbum. Preescolar. Primer grado. México, D. F.: Autor.

Secretaría de Educación Pública. (2014c). Mi álbum. Preescolar. Segundo grado. México, D. F.: Autor.

Secretaría de Educación Pública. (2014d). Mi álbum. Preescolar. Tercer grado. México, D. F.: Autor.

Siegler, R. S. (2009). Improving the numerical understanding of children from low-income families. Child Development Perspectives, 3(2), 118-124.

Steffe, L. P., \& Cobb, P. (1988). Construction of arithmetical meanings and strategies. New York: Springer-Verlag.

JOSÉ LUIS CORTINA MORFÍN

Domicilio: Carretera al Ajusco \#24, Col. Héroes de Padierna, Tlalpan, Ciudad de México, C.P. 14200. Área académica 2.

Teléfono: $\quad 56309700$ 